\title{
Research on the Deformation and Aerodynamic Characteristics of Flexible Flapping Wings Mimicking Cicada's Wing ZHANG Xijin ${ }^{1, a}$, DONG Qiang ${ }^{1, b}$, ZHAO Ning $^{1, c}$, SHEN Yuan ${ }^{1, d}$, WANG $\mathrm{Yan}^{2, \mathrm{e}}$
}

\author{
${ }^{1}$ School of Mechatronics, Northwestern Polytechnical University, China; \\ ${ }^{2}$ Xi'an Technological University North Institute of Information Engineering, China \\ a zhangxijin@nwpu.edu.cn, bstrongdq@aliyun.com, czhaon@nwpu.edu.cn, d624151991@qq.com, \\ e1023293832@qq.com
}

Keywords:Fluid-structure coupling, Flexible flapping wing, Lift and thrust coefficient, ANSYS

\begin{abstract}
Flexibility of an insect wing has an important effect on the aerodynamic characteristics. In this paper, deformations and aerodynamic characteristics of flexible flapping wings referred to a real cicada's wing are investigated. Firstly, 3 flexible flapping wing models referred to a real cicada's wing are established. Then, a method of fluid-structure coupling for flexible flapping wings is studied in ANSYS. Finally, deformations as well as lift \& drag characteristics of the 3 flexible flapping wings are simulated with the method of fluid-structure coupling. The result indicates that deformations of flexible flapping wings can enhance the aerodynamic performances, and the model whose flexibility is similar to that of a real cicada's wing has better aerodynamic characteristics.
\end{abstract}

\section{Introduction}

Flapping-wing micro aerial vehicles (FMAV) has a wide application prospect, it has become a research focus in the area of micro air vehicle (MAV) ${ }^{[1]}$. FMAV can be grouped into insect-like and bird-like FMAV. Because bird-like FMAV involves lower frequency and bigger size, it can be easily developed, and many bird-like FMAV have been created successfully. Caltech Micromachining Laboratory, UCLA and AeroVironment Inc. built the microbat that has demonstrated successful free flights with flight duration ranges from 5 to 18 seconds $^{[2]}$. Researchers at Delft University of Technology in the Netherlands have created the DelflyII which is a $30 \mathrm{~cm}$ device and can fly horizontally at $15 \mathrm{~m} / \mathrm{s}^{[3]}$. SRI and Toronto University developed "Mentor" whose wingspan is $15 \mathrm{~cm}^{[4]}$. Krashanitsa et al. developed a 74-cm-wing-span ornithopter equipped with an automatic flight control system. ${ }^{[5]}$ On the insect scale, Robert Wood at Harvard has used MFI technology to achieve takeoff of a tethered $60 \mathrm{mg}$ flapping vehicle ${ }^{[6]}$. In all, insect-like FMAV is rare.

The Cicada is one of the largest insects ${ }^{[7-8]}$, so the FMAV mimicking cicada is easy to develop under the current technical level. Because the research work on FMAV mimicking cicada is still rare, our target is to develop a FMAV mimicking cicada, and the paper investigates the deformations and aerodynamic characteristics of flexible flapping wings referred to a real cicada's wing.

\section{Establishing flexible flapping wing models}

A real cicada wing is photographed using high performance digital camera, then the vector image is imported to Pro/E, and the edge contour of the cicada's wing is drawn, eventually a three-dimensional simplified model of the membrane is gotten( with uniform thickness of 100um). Next, only four veins are designed to facilitate the manufacture of the wing. Finally, carbon fiber composite and polyimide are selected as the materials of veins and membranes, respectively. The whole process of establishing a flexible wings model is shown as Fig. 1. 
Flexibility of a flapping wing has influence on aerodynamic characteristics, so we designed 3 types of flexible flapping wing models to analyze the influence. The 3 flexible flapping wings have the same membrane and same arrangement of veins, while their vein sizes are different, so they are of different flexibilities. Point A and point B are selected as testing points. Point A is located on the fore vein, so its movement can indicate the flexibility of fore vein, while point $B$ is located on the inner vein, and its movement can indicate the flexibility of inner wing. When vertical loading of lgram is applied to point $A$ and point $B$ respectively, deformation of the 3 types of wing model is shown in Table 1. Among the 3 models, model 1 has the similar flexibility as the real cicada's wing.
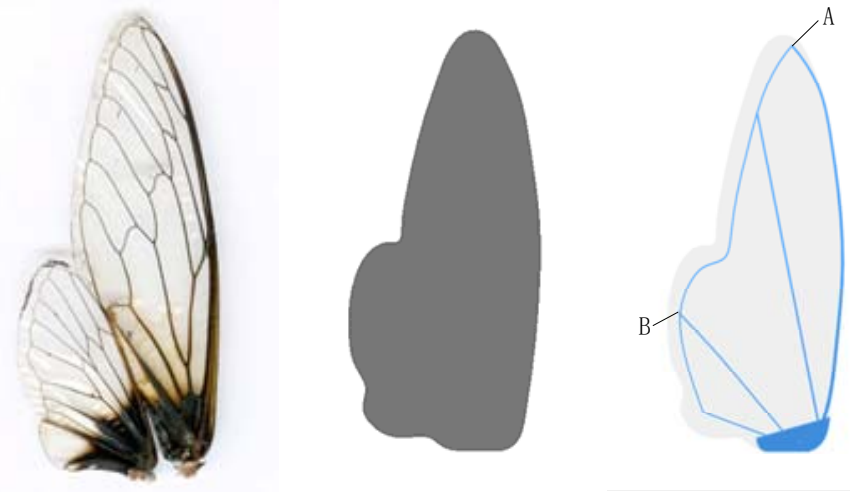

Fig. 1 The whole process of establishing a flexible flapping wing

Table 1: Deformation of 3 flexible wing models and rigid wing (unit: $\mathrm{mm}$ )

\begin{tabular}{c|c|c|c}
\hline testing point & model 1 & model 2 & model 3 \\
\hline A & 0.752 & 0.712 & 3.623 \\
\hline B & 0.824 & 0.410 & 1.460 \\
\hline
\end{tabular}

\section{The method of fluid-structure coupling for flexible flapping wings}

\subsection{The model of Fluid-structure Coupling for flexible flapping wings}

A cuboid of $600 \mathrm{~mm} \times 300 \mathrm{~mm} \times 600 \mathrm{~mm}$ is regard as fluid computational domain, and surface of membrane is chosen as the fluid-structure interaction interface. In this way, structural characteristics of membrane and vein are all considered in structural computation, only deformation and movement of the membrane is transferred to fluid computational domain. In Fluent, the shape of membrane is updated through dynamic grid technique, and the force on the surface of membrane is calculated, then the force is transferred to structural computation. Finally, the model is imported to Transient Structural and Fluent respectively, and the Transient Structural and Fluent are connected by System Coupling, thus the fluid-structure coupling can be done.

\subsection{Setting calculation parameters}

\subsubsection{Setting structural calculation parameters}

In the Transient Structural main interface, the material parameters of membrane and vein are set. The model of fluid domain is suppressed. Meshing method and size of membrane surface is set, and unstructured triangular grid is applied to membrane surface. An axis of reference is set, and its 2 rotary motions around a fixed coordinate system stand for flapping motion and twist motion of wing. The rotary rate is based on the reference 8. The membrane surface is set as Fluid Solid Interface. Time Step is $0.0001 \mathrm{~s}$, Step End Time is $0.05 \mathrm{~s}$, and Large Deflection is on. The calculation parameters are transferred to System Coupling. 


\subsubsection{Setting flow calculation parameters}

Meshing method and size of membrane surface in Fluent is as same as in Transient Structural, membrane surface is also set as system coupling interface, such parameters as end time, step size, etc. are as same as in in structural calculation, The calculation parameters in flow field are transferred to System Coupling.

\subsubsection{Setting coupling calculation parameters}

Analysis Type is set as Transient, solving method is sequential coupling. Because the wing motion brings change of flow field, Transient Structural is set as the first in order, and Fluent is set as the second in order. The maximum and minimum iterations are 10 and 1. Fluid Solid Interface in structure calculation and the System Coupling in flow field calculation are all chosen. Right-clicking is to create a data transfer, RMS Convergence Target in data transfer control is set as 0.01. The right Step Interval of Intermediate Restart Data Output is chosen. Finally, coupling calculation began with clicking the option of update.

\section{Deformation and aerodynamic characteristics of flexible flapping wings mimicking cicada}

\subsection{Deformation of flexible flapping wings mimicking cicada}

Model 1 was taken as an example to illustrate the process of analysis. Under the condition of 40 $\mathrm{Hz}$ flapping frequency, $15^{\circ}$ twist degree, $45^{\circ}$ flapping amplitude and $5 \mathrm{~m} / \mathrm{s}$ flow velocity, coupling calculation with 500 steps is finished. The second cycle is a stable situation, so data in the second cycle are analyzed. Deformation of the flexible wing mimicking cicada in the flapping process is shown in Fig. 2.

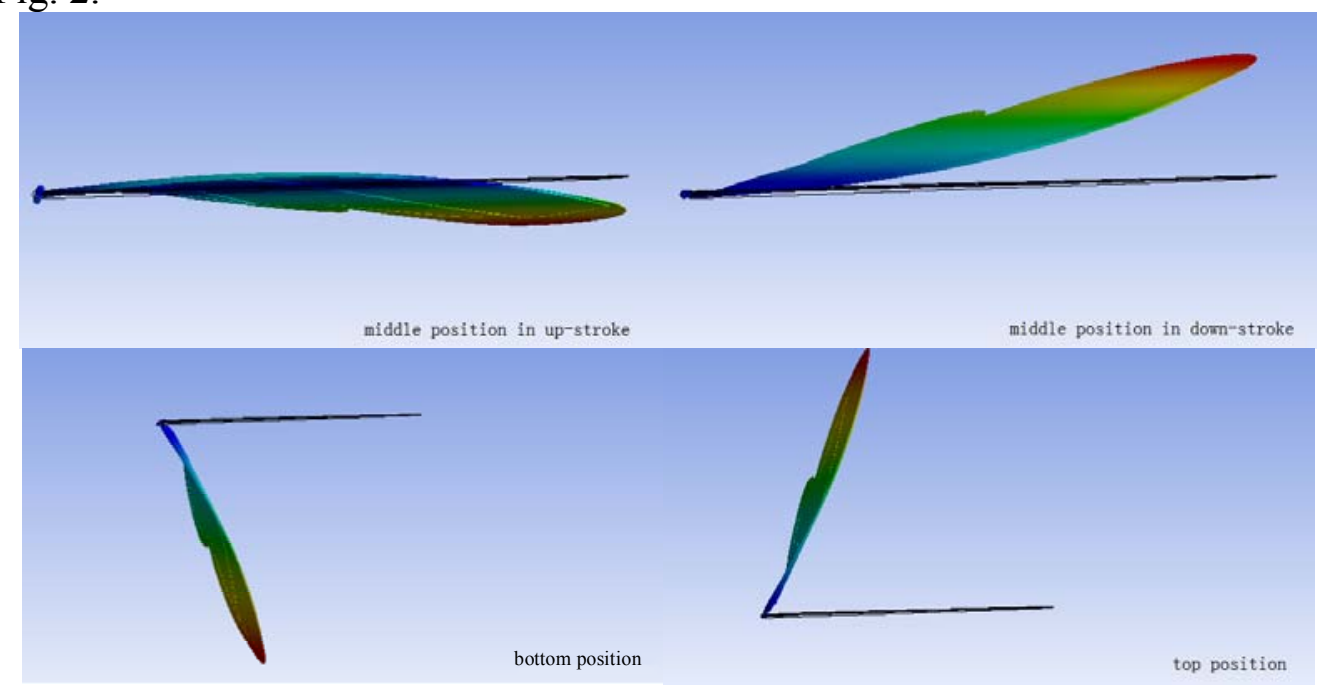

Fig. 2 Deformation of the flexible wing mimicking cicada in the flapping process

From Fig. 2 it is indicate that obvious spanwise and chordwise deformation appear in in the middle position. And the amount and direction of deformation vary with position of the wing. The variation has an important influence on characteristics of the wing.

For further comparative analysis of 3 models, point A and point B (as shown in Fig. 1) are taken as testing points. Their displacements are tested. To compare flexible wing with rigid wing, we subtract displacements of point $\mathrm{A}$ and point $\mathrm{B}$ in rigid wing from that of corresponding points in each model. Displacement differences of point A and point B is shown as Fig. 3 and Fig. 4.1 on the horizontal axis in Fig. 3 and Fig. 4 stands for a cycle, so the time-span from 0 to 0.25 is the period of wing motion from bottom position to middle position in up stroke. That from 0.25 to 0.5 is the period of wing motion from middle position to top position in up stroke, that from 0.5 to 0.75 is the period of wing motion from top position to middle position in down stroke, and that from 0.75 to 1 
is the period of wing motion from middle position to bottom position in down stroke. To some extent, deformations (or displacement differences) indicates bending and twisting of wing.

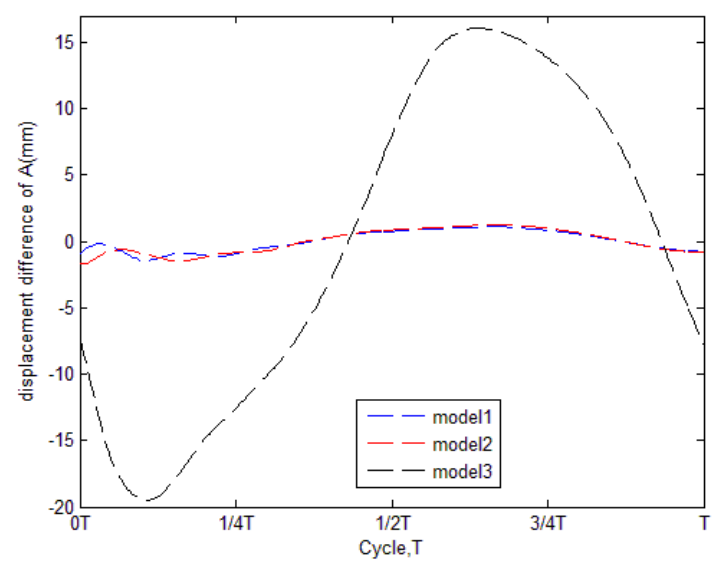

Fig. 3 Displacement differences of point A

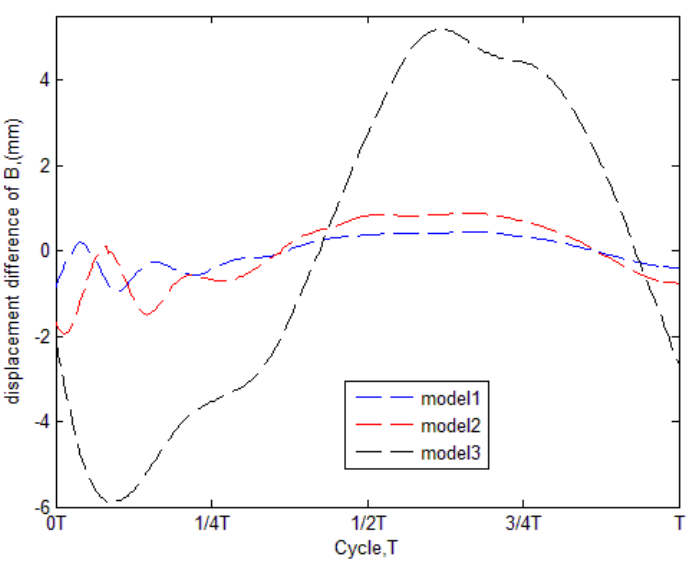

Fig. 4 Displacement differences of point B

From Fig. 3, it is indicates the mode 3 has the largest bending deformation, and the largest bending deformation is near to the bottom position. From Fig. 4, it is indicates the mode 3 also has the largest torsion deformation, and the largest torsion deformation appear near the bottom position.

\subsection{Aerodynamic characteristics of flexible flapping wings mimicking cicada}

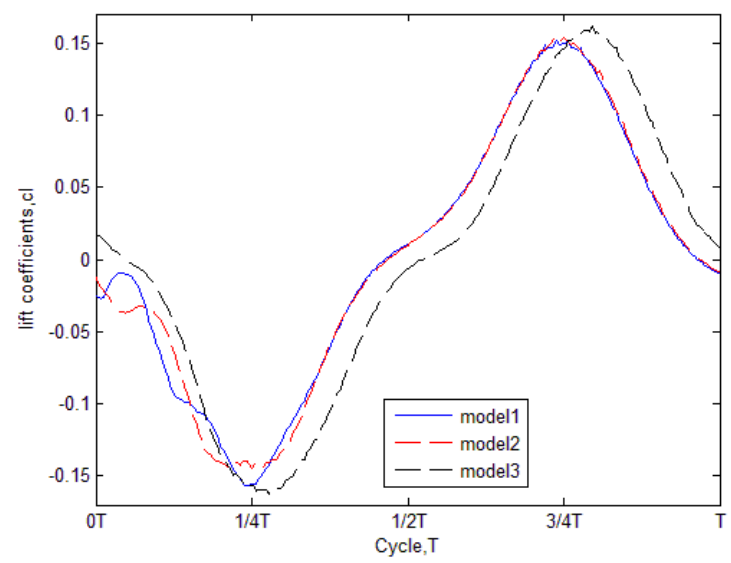

Fig. 5 The comparison of lift coefficients

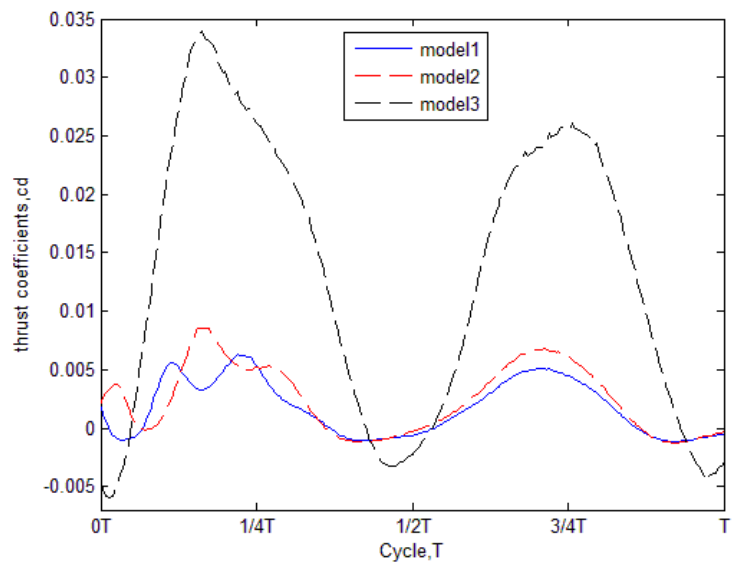

Fig. 6 The comparison of thrust coefficients

The comparison of lift coefficients is shown in Fig. 5. it indicates that flexibility has a large effect on lift coefficient, and lift coefficients change is complex. The comparison of thrust coefficients is shown in Fig. 6. For the detailed comparison, we calculate the mean lift coefficient and thrust coefficient (as shown in Table 2). The comparison result shows that model 1 has the best aerodynamic characteristics, and its mean lift coefficient and thrust coefficient are all large. The result also shows that insect wing has appropriate flexibility, and it produce the best aerodynamic characteristics.

Table 2: The comparison the mean lift coefficient and thrust coefficient

\begin{tabular}{c|c|c|c}
\hline Coefficient & model 1 & model 2 & model 3 \\
\hline mean thrust coefficient & 0.0017 & 0.0025 & 0.0132 \\
\hline mean lift coefficient & 0.000312 & 0.000187 & -0.00068 \\
\hline
\end{tabular}




\section{Conclusion}

In this paper, cicada's wings is regard as a bionic object, 3 types of flexible flapping wing models are designed. The method of fluid-structure coupling for flexible flapping wings is researched. And based on fluid-structure coupling computing, deformations and aerodynamic characteristics of the 3 flexible flapping wings are investigated. The result shows that mode 11 whose flexibility is similar to that of a real cicada's wing can produce the better aerodynamic characteristics.

\section{Acknowledgements}

This work is supported under National Natural Science Foundation of China (51175426), Natural Science Basic Research Plan in Shaanxi Province of China (2012JM7010) and the Key Laboratory of Road Construction Technology and Equipment (Chang' an University), MOE (2013G1502050). The authors gratefully acknowledge these financial supports.

\section{References}

[1] Dickinson, M.H., Lehmann, F.O., Sane, S.P. Wing rotation and the aerodynamics basis of insect flight. Science, 1999, 284, 1954-1960 .

[2] PORNSIN-Sirirak T N, LEE S W, NASSEF H, et al. MEMS wing technology for a batterypowered ornithopter [A]. In: The Thirteenth Annual International Conference on Micro Electro Mechanical Systems[C]. Miyazaki, Japan, 2000, 2: 799-804.

[3] Erik Edward Steltz, Redesign of the Micromechanical Flying Insect in a Power Density Context[D], 2008, University of California at Berkeley.

[4] SRI. Artificial muscle transducers[EB/OL]. http://www.erg.sri.com/automation/actuators. html. 2004-10-10.

[5] R.Y. Krashanitsa, D. Silin, S.V. Shkarayev, G. Abate. Flight dynamics of a flapping-wing air vehicle, Int. J. Micro Air Veh., 2009, 1(1): 35-49.

[6] Robert J. Wood. The First Takeoff of a Biologically Inspired At-Scale Robotic Insect, IEEE Transactions on Robotics, April 2008, vol. 24, no. 2, 341-347.

[7] F. Song, K. L. Lee, A. K. Soh, F. Zhu and Y. L. Bai. Experimental studies of the material properties of the forewing of cicada. The Journal of Experimental Biology 2004, 207, 3035-3042.

[8] Zhang Xi-Jin, Huang Qiang-Qiang, Luo Xi, et al. Research on the wing kinematics and bionic model of cicada. Applied Mechanics and Materials, 2013, 419: 17-22. 